\title{
REFLECTION OF RAYLEIGH WAVES FROM A RESONANT ELEMENT
}

\author{
共鳴体からのレイリー波反射 \\ V.P. Plessky and A.W. Simonian \\ Institute of Radioengineering and Electronics \\ Acad. of Sci. USSR \\ (Received 27, November 1990)
}

\begin{abstract}
Rayleigh wave scattering on a resonant element is discussed. As a resonator model a small load supported by a spring was chosen. It is shown, that near the eigenfrequency of resonator the reflection coefficient may reach 0.5 , which allows to design wide-band local mirrors for the Rayleigh waves.
\end{abstract}

Key words: Rayleigh wave, acoustoelectronic devices, surface acoustic wave, Bragg reflector.

\section{Introduction}

The Rayleigh waves find wide application in acoustoelectronic devices. In such devices frequently arises the necessity to reflect the wave. By now broadband "mirrors" for this purpose have not been found. The surface acoustic wave (SAW) reflection is usually realized using a distributed Bragg reflector, which is a periodic system of elements, each of which reflects the wave but very weakly though the total reflection may be almost complete. Such reflectors are narrow-band and effectively reflect the wave in the Bragg stop-band frequencies $\Delta f f f_{0} \approx r \ll 1$ (or $\Delta f f f_{0} \approx N^{-1}$ at $N r \ll 1$ ), where $\Delta f$ is the stop-band of the grating, $f_{0}=v_{R} / 2 l$ is the Bragg frequency ( $l$ is the structure period and $v_{R}$ is the SAW velocity), $r$ is the reflection coefficient of the element and $N$ is the number of the structure elements.
Distributed reflectors find wide applications in narrow-band filters, resonators and other SAW devices. Wide application is also envisaged for broad-band localized SAW reflectors, which might be used similarly to mirrors (including semi-transparent) in optical systems.

In the present paper we show, that the strong broad-band Rayleigh wave reflection from a single localized element may be realized provided this element is a resonator, i.e. processes eigenmodes of oscillations, exited by the incident wave. In practice such "acoustic surface states" may set in, as it was recently demonstrated by A.A.Maradudin, ${ }^{1}$ if the soundguide surface has protuberance with dimensions comparable to the wavelength. In a work by Japanese authors $^{2}$ a suggestion is made to use reflecting elements 
with interior degrees of freedom (Fig. 1a). In such elements, according to the authors, the "energy storage effects $^{13}$ are especially pronounced which leads to a more effective reflection. Experimentally a reflection coefficient from a single element of $r \approx 0.1$ has been obtained.

\section{Results and Discussion}

We shall consider reflection of a Rayleigh wave propagating along the surface of an isotropic soundguide from a single basic reflector having a resonance eigenfrequency (Fig. 1b). Let us take a basic resonator model which will be a small load of mass $m(\mathrm{~g} / \mathrm{cm})$ supported by a spring, having the stiffness $k$ (also for a unit length of the reflector along the $O Z$ axis). We assume that the eigenfrequency of the resonator $\omega_{0}$ is comparable with the frequency $\omega$ of the waves being scattered. We also assume that the spring responses only to vertical displacements of the soundguide surface at the point $x=0$ and the pressure exerted by the spring on the soundguide surface is applied uniformly over a narrow strip zone $|x| \leq a$, where $a$ « $\lambda / 2$.

The resonator oscillations in our model are described by equation $m \ddot{y}=-k(y-u)$, where $y$ is the load displacement, $u$ is the vertical displacement of the soundguide surface at the point $x=0,\left(u=u_{0} e^{-i \omega t}\right)$. Solving this equation we find the force $F$ exerted by the resonator on the soundguide:

$$
F=k \cdot \frac{\omega^{2}}{\omega_{0}^{2}-\omega^{2}} u_{0} \cdot e^{-i \omega t}
$$

The movement of the isotropic elastic medium will be described introducing the scaler $\varphi$ and vector $\vec{\psi}$ potentials, i.e. considering the displacement $\vec{u}$ to be equal to $\vec{u}=\operatorname{grad} \varphi+\operatorname{rot} \vec{\psi}$ where the potentials satisfy the equations $\Delta \varphi+k_{l}^{2} \varphi=0, \quad \Delta \psi+k_{t}^{2} \psi=0$ (where $k_{l}^{2}=\omega^{2} \rho /(\lambda+2 \mu) P, \quad k_{t}^{2}=\omega^{2} \rho / \mu \quad$ are the wavenumbers of the longitudinal and transverse bulk waves, $\rho$ is the body density and $\lambda$ and $\mu$ are the elasticity constants). We shall write the solutions of the motion equations in the form:

$$
\begin{aligned}
& \varphi=\varphi_{0} e^{p_{0} y+i q_{0} x}+\int_{-\infty}^{+\infty} \varphi(q) e^{p y+i q x} d q, \\
& \psi=\psi_{0} e^{s \theta^{y}+i q_{0} x}+\int_{-\infty}^{+\infty} \psi(q) e^{s y+i q x} d q .
\end{aligned}
$$

(For two-dimensional motion $\vec{\psi}$ may be written as $\vec{\psi}$ $=(0,0, \psi))$. Here $\varphi_{0}$ and $\psi_{0}$ are partial amplitudes of the Rayleigh wave incident on the resonator, $p=$ $\sqrt{q^{2}-k_{l}^{2}}$ and $s=\sqrt{q^{2}-k_{t}^{2}}$; the same quantities with the subscript "0" satisfy the Rayleigh equation $4 p_{0} s_{0} q_{0}^{2}=\left(q_{0}^{2}+p_{0}^{2}\right)^{2}$. The amplitudes $\varphi(q)$ and $\psi(q)$ of the scattered waves are to be determined. Integration in (1) and (2) is carried out along the real axis, with the singular points on the positive semiaxis being circumvented from below and those on the negative one from top. In this, if $|q|>k_{t}$ positive $p$ and $s$ values are chosen, if $|q|<k_{t}$ then $s=-i / s /$ and, correspondingly, at $|q|<k_{l} \quad p=-i|p|$. Such choice of the complex functions $p$ and $s$ branches and the integration path ensures decay of the oscillations towards the interior of the soundguide (or wave radiation from the resonator towards the inside of the soundguide).

The resonator and soundguide interact through the surface of the solid at the point $x=0$. We assume that the resonator creates no horizontal stresses, i.e. $\sigma_{x y}=0$ at $y=0$ for all $x$ points. From this we find that $\psi(q)=$ $-\frac{2 i q p}{q^{2}+s^{2}} \cdot \varphi(q)$, this relation being also valid for quantities with the subscript "0", that describe the 


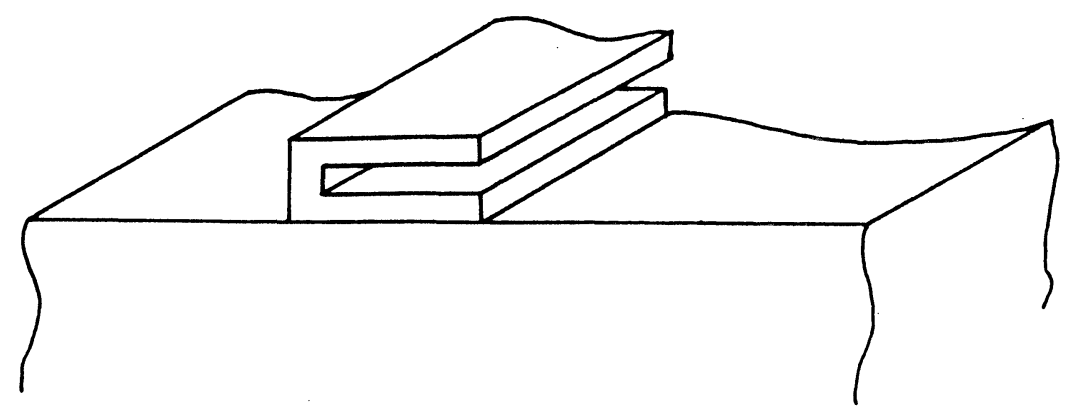

a

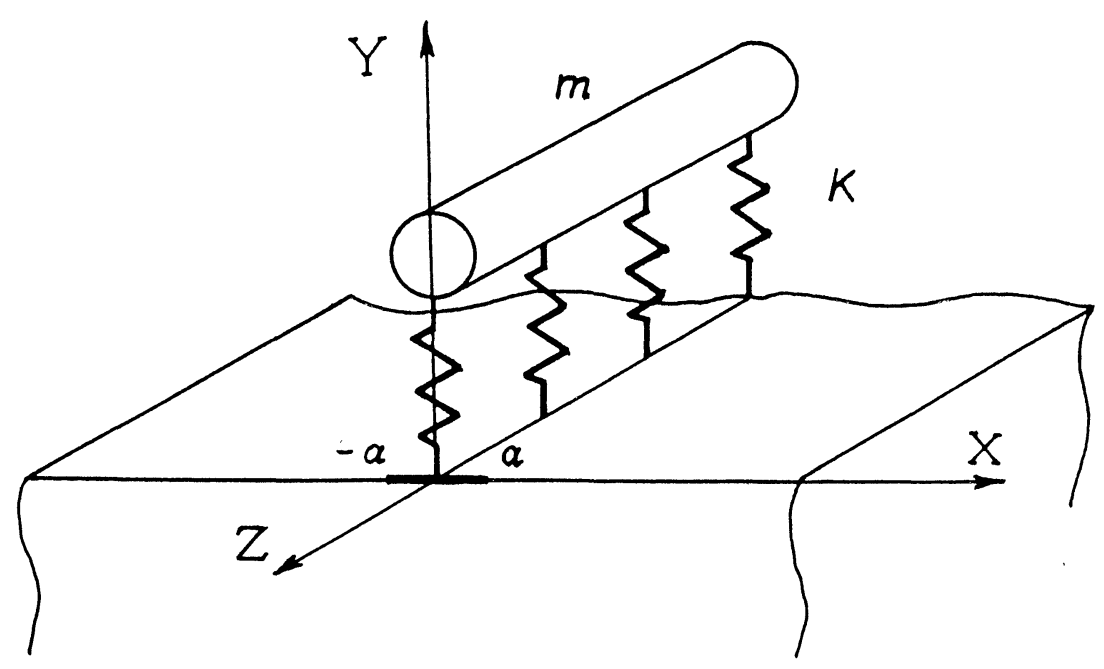

$\mathrm{b}$

Fig.1. a) Reflecting element with interior degrees of freedom. b) The resonator model.

incident Rayleigh wave. Normal stresses in the $|x|<$ $a$ region are defined from the force $F$ exerted by the resonator: $\sigma_{y y} /_{y=0}=12 a$. Expressing the quantities included in this equation in terms of potentials according to the known formulae we obtain integral equation for the amplitudes $\varphi(q)$ of the waves being scattered:

$$
\begin{gathered}
\mu \int_{-\infty}^{+\infty} \frac{4 q^{2} p s-\left(q^{2}+s^{2}\right)^{2}}{q^{2}+s^{2}} \varphi(q) e^{i q x} d q \\
=\frac{k}{2 a} \frac{\omega^{2}}{\omega_{0}^{2}-\omega^{2}} \frac{p_{0} k_{t}^{2}}{q_{0}^{2}+s_{0}^{2}} \varphi_{0} \\
\quad+\int_{-\infty}^{+\infty} \frac{p k_{t}^{2}}{q^{2}+s^{2}} \varphi(q) d p \Pi(x),
\end{gathered}
$$

where $\Pi(x)=1$ at $|x|<a$ and $\Pi(x)=0$ at $|x|>a$. 
Representing $\Pi(x)$ in the form $\Pi(x)=\int_{-\infty}^{+\infty} \Pi(q) e^{i q x} d q$ we rewrite eq. (3) as

$$
\begin{gathered}
\frac{D(q)}{k_{t}^{2}\left(q^{2}+s^{2}\right)} \varphi(q)=\varepsilon \cdot \frac{\omega_{0}^{2}}{\omega_{0}^{2}-\omega^{2}}\left\{\frac{p_{0} k_{t}}{q_{0}^{2}+s_{0}^{2}} \varphi_{0}\right. \\
+\int_{-\infty}^{+\infty} \frac{p k_{t}}{q^{2}+s^{2}} \varphi(q) d q M(q),
\end{gathered}
$$

where the Rayleigh determinant $D(q) \equiv 4 q^{2} p s-\left(q^{2}+\right.$ $\left.s^{2}\right)^{2}$ and the parameter $\varepsilon=\pi \frac{m}{\rho a \lambda} \quad$ are introduced.

The physical sense of $\varepsilon$ is quite clear, i.e. the linear mass $m$ is compared to the soundguide mass under the strip zone $2 a$ at the depth $\lambda / 2 \pi$.

The solution of eq.(4) is easy. It should be noted that the expression in the braces on the right-hand side of eq.(4) is a certain number $\Phi$ (functional). We designate $\Phi(\omega)=\frac{\omega_{0}^{2}}{\omega_{0}^{2}-\omega^{2}} \cdot \Phi$ and from eq.(4)

directly obtain:

$$
\varphi(q)=\varepsilon \Phi(\omega) \cdot \frac{\Pi(q) k_{t}^{2}\left(q^{2}+s^{2}\right)}{D(q)} .
$$

Using this expression and the definition of $\Phi$ we find that

$$
\Phi(\omega)=\frac{p_{0} k_{t} /\left(q_{0}^{2}+s_{0}^{2}\right)}{1-\frac{\omega^{2}}{\omega_{0}^{2}}-\varepsilon \cdot \int_{-\infty}^{+\infty} \frac{p k_{t}^{3}}{D(q)} \Pi(q) d q} \cdot \varphi_{0} .
$$

Equations (5) and (6) solve the problem. The scattered waves are found by integration of eq.(5): $\varpi(x)=$ $\int_{-\infty}^{+\infty} \varphi(q) e^{i q x} d q$, or

$$
\varphi(x)=\varepsilon \Phi(\omega) \cdot \int_{-\infty}^{+\infty} \frac{k_{t}^{2}\left(q^{2}+s^{2}\right)}{D(q)} \Pi(q) e^{i q x} d q .
$$

In the present paper we confine ourselves to finding the reflection coefficient of the Rayleigh wave. It can be done by the calculating the residue at the point $q=-q_{0}$ in the integral of eq.(7):

$$
R=-2 i \pi \cdot \frac{\varepsilon p_{0} \Pi\left[-q_{0}\right]}{1-\frac{\omega^{2}}{\omega_{0}^{2}}-\varepsilon I} \cdot \frac{k_{t}^{3}}{\left.D_{q}^{\prime}\right|_{q}=-q_{0}},
$$

where designation $I=\int_{-\infty}^{+\infty} \frac{p k_{t}^{3}}{D(q)} \Pi(q) d q$ is introduced and $\Pi(q)=\sin q a /(\pi q)$. The $I$ value depends on the Poisson coefficient $\sigma$, the width of the strip zone $a / \lambda_{0}$ and the relative frequency of the incident wave $\omega / \omega_{0}\left(\lambda_{0}=2 \pi v_{R} / \omega_{0}\right)$. The parameter $\varepsilon$ also depends on the frequency: $\varepsilon=\varepsilon_{0} \omega / \omega_{0}$, where $\varepsilon_{0}=\pi \cdot \frac{m}{\rho a \lambda_{0}}$. The integral $I\left[a, \frac{\omega}{\omega_{0}}\right]$ was found numerically. Figure 2 shows the real and imaginary parts of $I$ for $a=\lambda_{0} / 16$. Figure 3 shows $R\left(\omega / \omega_{0}\right)$ vs frequency for several $\varepsilon$ values. It is interesting to note that the resonant frequency is lower than $\omega_{0}$, i.e. the soundguide portion, to which the resonator is attached, plays the role of an additional spring as if lessening the resonator spring stiffness. The maximum value of the reflection coefficient $R_{\max }$ is reached at the resonant point and is $\left|R_{\max }\right|$ $\approx 0.5$ at a Poisson coefficient of $\sigma=0.17$. At $\varepsilon_{0} » 1$ effective reflection takes place over a broad band of frequencies above the resonance frequency.

\section{Summary}

Thus we have demonstrated that resonant reflecting elements may be used as effective broad-band mirrors 


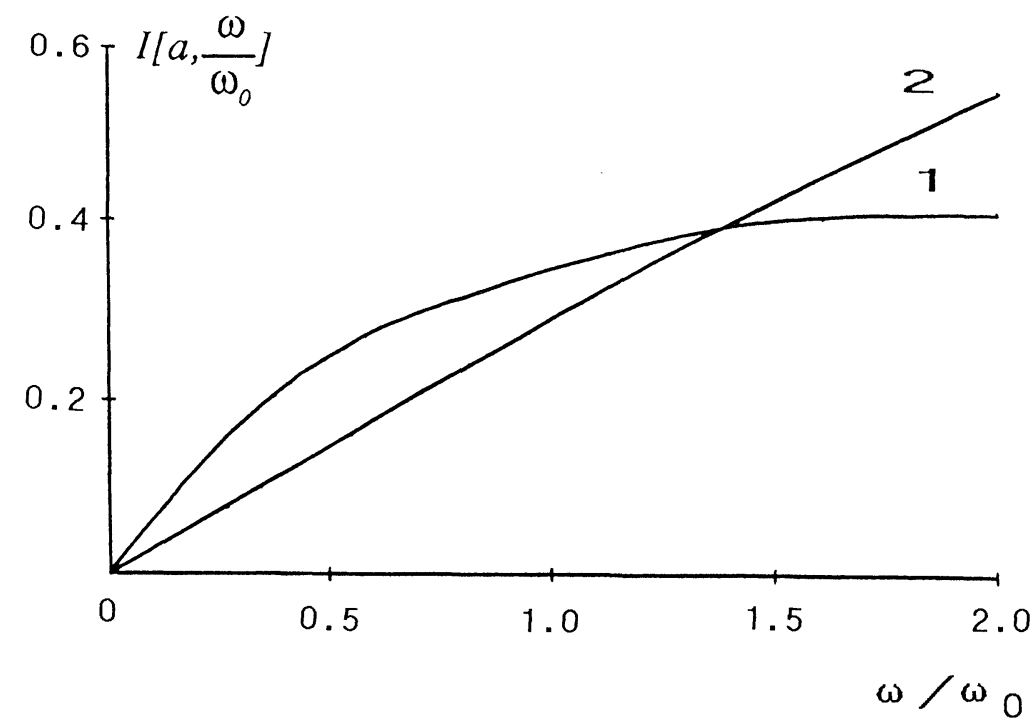

Fig.2. The real (1) and imaginary (2) parts of $I$ for $a=\lambda_{0} / 16$ vs frequency.

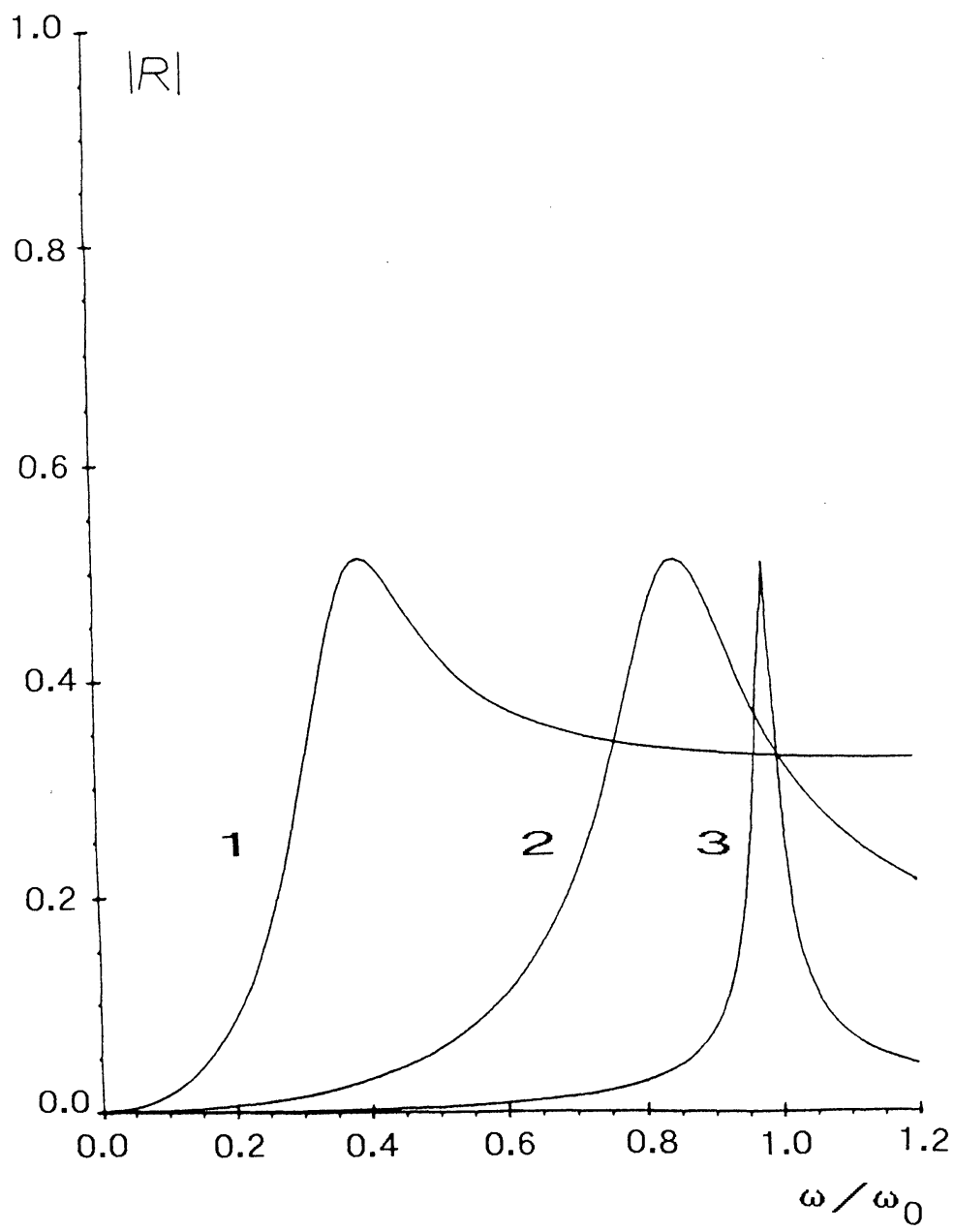

Fig.3. The frequency relationship of $R$ for $a=\lambda_{0} / 16$ : $1-\varepsilon_{0}=10 ; 2-\varepsilon_{0}=1 ; 3-\varepsilon_{0}=0.1$. 
for surface acoustic Rayleigh waves.

The authors express their thanks to Yu.V.Gylyayev and V.I.Grigoryevskii for the discussion of the work and to E.A.Garova for her help in carrying out the numerical calculations.

\section{REFERENCES}

${ }^{1}$ A.A. Maradudin, A.R. McGurn, Phys. Rev. B 39, 87328735(1989)

${ }^{2}$ K. Yamanouchi, T. Meguro and Z.H. Chen, Proc. IEEE Ultrasonics Symp., 173-176(1987).

${ }^{3}$ R.C.M. Li, J. Melngailis, IEEE Trans. on Sonics and Ultrasonics SU-22, 189-198 (1975).

This paper was presented in the Japan-USSR Joint Symposium at Moscow, Oct. 22, 23, 1990. 